Colloids and Surfaces

A: Physicochemical and Engineering Aspects 167 (2000) 165-177

\title{
Micelle formation and CMC of gemini surfactants: a thermodynamic model
}

\author{
Terri A. Camesano ${ }^{a, *}$, R. Nagarajan ${ }^{b}$ \\ ${ }^{a}$ Department of Civil and Environmental Engineering, Pennsylvania State University, 212 Sackett Building, University Park, \\ PA 16802-1408, USA \\ ${ }^{\mathrm{b}}$ Department of Chemical Engineering, Pennsylvania State University, 161 Fenske Laboratory, University Park, PA 16802, USA
}

\begin{abstract}
Gemini surfactants possess a structure resembling a pair of conventional single chain surfactants covalently connected by a spacer. The spacer can vary in length and chemical structure. In this paper, the aggregation behavior of gemini surfactants is examined on the basis of a free energy model developed by extending our theory for conventional surfactants. Free energy contributions beyond those considered for conventional surfactants arise because of the spacer. They originate from the fact that (a) the spacer shields the hydrophobic core of the aggregate from contact with water, (b) the spacer constrains the distance between the head groups, thus imposing non-uniformity in charge distribution at the aggregate surface, (c) the spacer prevents the two linked tails from having a packing conformation inside the micelle identical to that of analogous unlinked single chain surfactants, and (d) the spacer can be partially buried inside the micelle core provided its length and the molecular interactions allow it. Illustrative calculations for gemini surfactants with tail lengths of 5-16 carbon atoms and various spacers are discussed here. The spacers considered include the polymethylene chain and a combination of polymethylene chain with $\mathrm{NCH}_{3}, \mathrm{O}, \mathrm{S}$, and aromatic groups. The predicted critical micelle concentration (CMC), micelle aggregation number, sphere-to-rod transition parameter $(K)$ and shape of aggregates are compared with available experimental data. A comparative analysis of the magnitude and shape dependence of the individual contributions to the free energy shows that the nonuniform charge distribution at the aggregate surface and the additional packing constraint on the tails, both originating from the short length of the spacer, play a central role in determining the equilibrium shape of the aggregates. (C) 2000 Elsevier Science B.V. All rights reserved.
\end{abstract}

Keywords: Micelle formation; Gemini surfactants; Thermodynamic model

* Corresponding author. Tel.: + 1-814-865-4851; fax: + 1814-863-7304.

E-mail address: terric@psu.edu (T.A. Camesano)

\section{Introduction}

A gemini surfactant is a molecule composed of two identical hydrophilic head groups and hydrophobic tail groups, equivalent to two single chain surfactants, that are covalently connected by a spacer. The spacer can vary in length and chemi- 
cal structure, be rigid or flexible, and be hydrophobic or hydrophilic. The term 'gemini surfactant' was first applied to molecules consisting of a hydrocarbon tail, an ionic group, a rigid spacer, a second ionic group, and another hydrocarbon tail [1]. However, molecules with non-rigid spacers and headgroups that are not ionic have also been synthesized and referred to as gemini or dimeric surfactants.

Gemini surfactants with quaternary ammonium bromide head groups and linear alkyl tails have been the most studied (c.f. papers by Zana et al.). These surfactants have the general formula $\quad\left[\mathrm{C}_{m} \mathrm{H}_{2 m+1}-\mathrm{N}^{+}-\left(\mathrm{CH}_{3}\right)_{2}-\left(\mathrm{CH}_{2}\right)_{S}-\left(\mathrm{CH}_{3}\right)_{2}-\right.$ $\left.\mathrm{N}^{+}-\mathrm{C}_{m} \mathrm{H}_{2 m+1}\right] 2 \mathrm{Br}^{-}$, and are referred to as $m-s-$ $m, 2 \mathrm{Br}^{-}$surfactants. Such molecules may be considered equivalent to the dimers of the monoquaternary ammonium bromide surfactants $\mathrm{C}_{m} \mathrm{H}_{2 m+1}-\left(\mathrm{C}_{S / 2} \mathrm{H}_{S+1}\right)-\mathrm{N}^{+}-\left(\mathrm{CH}_{3}\right)_{2} \mathrm{Br}^{-}$[2]. Besides these molecules with polymethylene group spacers, other geminis containing aromatic rings as spacers or with functional groups such as $\mathrm{S}, \mathrm{N}, \mathrm{O}$, or $\mathrm{NCH}_{3}$ in the polymethylene have also been studied in the literature [3-5].

The study of gemini surfactants has generated much interest due to their unusual solution and interfacial properties and their enhanced performance in applications, compared to analogous single chain surfactants (see most recent review in Ref. [6]). In particular, gemini surfactants have critical micelle concentrations (CMC) that are up to two orders-of-magnitude lower than the CMCs of corresponding single chain surfactants [7]. DTAB (dodecyl trimethyl ammonium bromide) can be considered the monomeric form of the $\mathrm{C}_{12}$ series of gemini surfactants, and has a CMC of $1.5-1.6 \times 10^{-2} \mathrm{M}[4,8]$. The gemini surfactant, $12-3-12,2 \mathrm{Br}^{-}$, has a CMC value of 9.1-9.6 $\times$ $10^{-4} \mathrm{M}[8,9]$. In addition, gemini surfactants of this series with longer spacers have even lower CMC values. For the molecule 12-16-12,2 $\mathrm{Br}^{-}$, $\mathrm{CMC}$ values of $1.2-1.4 \times 10^{-4}$ have been reported $[8,9]$, which are almost two orders of magnitude lower than the CMC values reported for DTAB. CTAB (cetyl trimethyl ammonium bromide) can be compared with the $\mathrm{C}_{16}$ series of gemini surfactants. The CMC for CTAB is $10^{-3}$ $\mathrm{M}$ [10], while for $16-3-16,2 \mathrm{Br}^{-}$, the $\mathrm{CMC}$ is nearly two orders of magnitude lower (2.50$\left.2.55 \times 10^{-5} \mathrm{M}\right)[8,11]$.

Further, gemini surfactants with short spacers form large, threadlike aggregates with interesting rheological properties, whereas the corresponding monomeric surfactant forms only small, spherical aggregates [12]. The micelle size and shape data have been obtained using a combination of cryoTEM imaging, time-resolved fluorescence quenching (TRFQ), and small-angle neutron scattering (SANS) experiments. Gemini surfactants with short spacers, 10-3-10,2 $\mathrm{Br}^{-}$and 12-3-12,2 $\mathrm{Br}^{-}$ form elongated rods or threadlike micelles at concentrations where the corresponding monomeric surfactant forms only spheres [12,13]. The gemini molecule 16-3-16,2 $\mathrm{Br}^{-}$also forms aggregates of lower curvature compared to its monomeric analog, as cryo-TEM imaging revealed vesicles, bilayer membrane fragments, and threadlike micelles [12].

The only theoretical investigation of gemini surfactants available in the literature pertains to their equilibrium behavior at the air-water interface $[14,15]$. In this study, the conformational entropy of the spacer chain was shown to play an important role in determining the equilibrium area per molecule. The model was able to describe qualitatively the non-monotonic behavior of the area per molecule at the air-water interface as a function of the spacer length for the 12-s-12,2 $\mathrm{Br}^{-}$ surfactants.

The main goal of this paper is to model the aggregation behavior of gemini surfactants in aqueous solutions and to explore the origin of their very low CMCs and their unusual ability to generate large micelles when the spacer length is small. For this purpose, we develop expressions for various contributions to the free energy of micellization of gemini surfactants by modifying our previous treatment of single chain ionic surfactants [16,17]. The modifications account for important spacer-related effects, namely, the presence of a part of the spacer at the micelle corewater interface, the non-uniform distribution of ionic head groups at the micelle surface, the packing constraint on the tails due to their connectivity via the spacer, and the possible burying of a part of the spacer inside the micelle core. 


\section{Thermodynamics of self-assembly}

The general thermodynamic principles of surfactant self-assembly are well established [16-19] and only the main results are mentioned below. The surfactant solution is viewed as a multi-component system consisting of water, singly dispersed surfactant molecules, and aggregates of all possible shapes and aggregation numbers, $g$. At equilibrium, the chemical potential of a surfactant in the singly dispersed state must equal the chemical potential per molecule of surfactant aggregates of any size and shape. For dilute solutions, this equilibrium condition can be represented as

$\mu_{g}^{\circ}+k T \ln X_{g}=g\left[\mu_{1}^{\circ}+k T \ln X_{1}\right]$

where $\mu_{g}^{\circ}$ is the standard state chemical potential of aggregate of size $g, X_{g}$ is its mole fraction in solution, and the subscript 1 refers to the singly dispersed surfactant. Eq. (1) can be rewritten in the form of the aggregate size distribution equation

$X_{g}=X_{1}^{g} \exp \left(-\frac{g \Delta \mu_{g}^{\circ}}{k T}\right)$

The factor $\Delta \mu_{g}^{\circ}\left(=\mu_{g}^{\circ} / g-\mu_{1}^{\circ}\right)$ denotes the change in the standard state chemical potential of a singly dispersed molecule when it is incorporated into an isolated micelle of size $g$.

The aggregate size distribution can be used to calculate all important solution properties of the surfactant system. There are several methods for determining CMC values [20]. Here, CMC is taken to be the value of $X_{1}$ at which the concentration of the singly dispersed surfactant is equal to the total amount of surfactant present in the aggregates, $X_{1}=\Sigma g X_{g}=X_{\mathrm{CMC}}$. From the size distribution equation, we can also calculate the average size of the aggregate and the size polydispersity at any monomer or total surfactant concentration.

The size distribution equation can be used to investigate the formation of all possible aggregate shapes. Small micelles tend to be spherical or globular. When large, elongated micelles form, they are envisioned to consist of spherical endcaps and a rodlike midsection. The standard state chemical potential of a surfactant molecule in the rodlike portion will be different from that of one in the endcap region. This standard state free energy difference is represented by the sphere-torod transition parameter, $K$, which measures the free energy advantage for a molecule to be in the cylindrical portion compared to being in the endcaps. Eq. (2) is recast for this case as

$X_{g}=\frac{1}{K}\left[X_{1} \exp \left(-\frac{\Delta \mu_{\mathrm{cyl}}^{\circ}}{k T}\right)\right]^{g}$,

$\ln K=g_{\text {cap }} \frac{\Delta \mu_{\text {cap }}^{\circ}-\Delta \mu_{\text {cyl }}^{\circ}}{k T}$

Here, $\Delta \mu_{\text {cyl }}^{\circ}$ is the difference in the standard chemical potential between a molecule in the cylindrical region compared to a singly dispersed molecule, the subscript 'cap' refers to the endcap region of the cylindrical micelle, and $g_{\text {cap }}$ molecules are located in the endcaps. The magnitude of $K$ determines whether the micelle is spherical or cylindrical, with $K$ values of $\sim 10^{7}-10^{12}$ being necessary for cylindrical micelles to form at physically realistic surfactant concentrations [19,21-25]. The number and weight-average aggregation numbers $\left(g_{\mathrm{n}}\right.$ and $\left.g_{\mathrm{w}}\right)$ of rodlike micelles are calculated at a total surfactant concentration $X$ using the relation $g_{\mathrm{n}}=g_{\text {cap }}+\left[K\left(X-X_{1}\right)\right]^{1 / 2}$,

$g_{\mathrm{w}}=g_{\text {cap }}+2\left[K\left(X-X_{1}\right)\right]^{1 / 2}$

In order to perform the size distribution calculations, an explicit expression for $\Delta \mu_{g}^{\circ}$ must be developed. This is done in terms of molecular properties, by considering all the physicochemical changes experienced by a singly dispersed surfactant when it gets incorporated into an isolated aggregate. The various contributions to the free energy of aggregation depend on the shape of the aggregates and hence, the geometrical properties describing possible aggregate shapes must be first defined. In this work, we consider spheres, globular shapes, cylindrical and endcap portions for rodlike micelles, spherical bilayer vesicles and planar bilayers. Expressions for aggregate volume, $V_{g}$, aggregate surface area, $A_{g}$, area per head group of the aggregate core, $a$, and aggregation number, $g$ for the shapes considered have been presented before [16,17]. To simplify computations, we have not performed the calculations for spherical bilayers but only for planar bilayers. We 
anticipate, however, that the free energy of aggregation would be close enough for these two shapes and the predicted formation of planar bilayers could suggest the possible formation of spherical vesicles at similar solution conditions.

\section{Expressions for various free energy contributions}

\subsection{Transfer free energy}

A negative contribution to the free energy of aggregation accompanies the transfer of the hydrophobic tails from water to the core of the micelle. The transfer free energy, $\left(\Delta \mu_{g}^{\circ}\right)_{\mathrm{tr}}$ depends on the temperature $T$, and the number of methyl and methylene groups in the tails. The group contributions of methyl and methylene groups to $\left(\Delta \mu_{g}^{\circ}\right)_{\mathrm{tr}}$ have been presented previously [17]

$$
\begin{aligned}
\frac{\left(\Delta \mu_{g}^{\circ}\right)_{\mathrm{tr}, \mathrm{CH}_{3}}=}{k T} & 3.38 \ln (T)+\frac{4064}{T}-44.23 \\
& +0.02595 T \\
\frac{\left(\Delta \mu_{g}^{\circ}\right)_{\mathrm{tr}, \mathrm{CH}_{2}}}{k T}= & 5.85 \ln (T)+\frac{896}{T}-36.15-0.0056 T
\end{aligned}
$$

For gemini surfactants, the contribution from both tails must be taken into account, as well as a contribution from the portion of the spacer buried within the core. The number of methylene units of the spacer buried in the micelle core is denoted by $s_{\text {core }}$. It is estimated as the difference between the total number of methylene groups in the spacer $s$ and the minimum number of groups $\left(=a^{1 / 2} / \ell_{\mathrm{CH}_{2}}\right)$ necessary to connect the two head groups separated by the distance $a^{1 / 2}, \ell_{\mathrm{CH}_{2}}$ being the length of a methylene group.

$s_{\text {core }}=s-\frac{a^{1 / 2}}{\ell_{\mathrm{CH}_{2}}}$

Obviously, $s_{\text {core }}=0$ when $s<a^{1 / 2} / \ell_{\mathrm{CH}_{2}}$.

For double chained molecules, intramolecular interactions between the chains exist even in their singly dispersed state. Therefore, for such molecules, a factor of 0.6 is used in estimating the transfer free energy of the second tail, consistent with the hydrocarbon solubility data available for some double chained molecules [18]. The same factor is used here also for the contribution from the buried part of the spacer. The total transfer free energy is thus calculated by including the contributions from the $(m-1)$ methylene groups and the terminal methyl group of each tail, and the buried methylene groups of the spacer.

$$
\begin{aligned}
\left(\Delta \mu_{g}^{\circ}\right)_{\mathrm{tr}}= & 1.6\left(\Delta \mu_{g}^{\circ}\right)_{\mathrm{tr}, \mathrm{CH}_{3}}+1.6(m-1)\left(\Delta \mu_{g}^{\circ}\right)_{\mathrm{tr}, \mathrm{CH}_{2}} \\
& +0.6 s_{\mathrm{core}}\left(\Delta \mu_{g}^{\circ}\right)_{\mathrm{tr}, \mathrm{CH}_{2}}
\end{aligned}
$$

\subsection{Interfacial free energy}

The free energy of formation of the micelle core-water interface $\left(\Delta \mu_{g}^{\circ}\right)_{\text {int }}$ is written as the product of the interfacial area and the aggregate core-water interfacial tension, $\sigma_{\text {agg }}$.

$\frac{\left(\Delta \mu_{g}^{\circ}\right)_{\text {int }}}{k T}=2 \frac{\sigma_{\mathrm{agg}}}{k T}\left(a-a_{o}\right)$

where $a$ is the equilibrium area of the aggregate core per headgroup and $a_{\mathrm{o}}$ is the area per tail shielded by the head group from contact with water. The factor 2 arises because there are two head groups per molecule and the free energy is expressed per molecule. The interfacial tension $\sigma_{\text {agg }}$ is taken equal to $\sigma_{\mathrm{sw}}$, the interfacial tension between water and the surfactant tail, and is calculated as described previously [16].

\subsection{Coverage free energy}

An additional coverage free energy arises since a part of the spacer is present at the micellar surface, replacing the micelle core-water contact by the micelle core-spacer contact. This extra coverage is a function of the spacer length, and is calculated by assuming a projected area for the spacer on the core surface

$\frac{\left(\Delta \mu_{g}^{\circ}\right)_{\text {cover }}}{k T}=\frac{\left(\sigma_{\mathrm{SP}}-\sigma_{\mathrm{agg}}\right)}{k T}\left[\left(s-2-s_{\text {core }}\right) \ell_{\mathrm{CH}_{2}} L\right]$

Here, $\sigma_{\mathrm{SP}}$ is the interfacial tension between the spacer and the micelle core $(=0$ for a polymethylene spacer $)$. The factor $\left(\sigma_{\mathrm{SP}}-\sigma_{\mathrm{agg}}\right)$ denotes the interfacial tension difference associated with the displacement of water by the spacer at the 
core surface. The factor $\left[\left(s-2-s_{\text {core }}\right) \ell_{\mathrm{CH}_{2}} L\right]$ represents the projected area of the spacer covering the micelle surface. Here, $L$ is the close-packed distance between polymethylene chains $(0.46 \mathrm{~nm})$, and $\left(s-2-s_{\text {core }}\right) \ell_{\mathrm{CH}_{2}}$ is the length of the polymethylene spacer (excluding the two terminal methylene groups which are taken to be part of the head group). Hence, the coverage effect is relevant only for $s>2$.

\subsection{Steric free energy}

Steric interactions account for the repulsion caused by crowding of the head groups at the micelle surface. Their magnitude has been estimated following the van der Waals approach $[16,26]$, as

$\frac{\left(\Delta \mu_{g}^{\circ}\right)_{\text {steric }}}{k T}=-\ln \left(1-\frac{a_{\mathrm{p}}}{a}\right)$

where $a_{\mathrm{p}}$ is the cross-sectional area of the head group near the surface of the micelle.

\subsection{Tail deformation free energy}

Surfactant tails stretch and deform so as to pack within the micelle core meeting liquid-like density constraints. A simple analytic expression for this free energy contribution has been developed before [16] using the approach employed for block copolymer microdomains [27]. For spherical micelles, the tail deformation free energy is

$\frac{\left(\Delta \mu_{g}^{\circ}\right)_{\mathrm{def}}}{k T}=2\left(\frac{9 P \pi^{2} R_{\mathrm{s}}^{2}}{80 N L^{2}}\right)$

where $P$ is the packing factor, $R_{\mathrm{s}}$ is the core radius, and $N$ is the number of segments in the tail. Taking 3.6 methylene units to constitute a segment of length $L$ (which is also equal to the close-packed distance between the polymethylene chains), $\quad N=(m+1) / 3.6$. For other aggregate shapes, the radius $R_{\mathrm{s}}$ is replaced by the cylinder radius $R_{\mathrm{c}}$ or by the half-bilayer thickness of the lamella $R_{\text {lam }}$, and the factor 9 is replaced by 10 for both cylinders and lamellae. The packing factor $P$ is equal to $1 / 3$ for spheres, $1 / 2$ for cylinders and 1 for lamellae [16]. The factor 2 in Eq. (11) arises because there are two tails per molecule.

\subsection{Packing free energy}

Eq. (11) is formulated for conventional surfactant molecules. In the case of gemini surfactants, when the spacer is short, it forces the two tails of the molecule to be too close to one another, assuming conformations different from those of two independent molecules unconnected by the spacer. Therefore, an additional packing free energy contribution beyond Eq. (11) is necessary to account for the constraint imposed by the spacer. We develop an expression for this free energy contribution as follows. Using the Semenov model for chain segment distribution as a function of the distance from the core-water interface $[16,27]$, one can calculate the area occupied by the methylene segments near the core-water interface. This effective area is

$a_{\text {eff }}=\frac{v_{\mathrm{o}}}{\eta \pi R}$

where $\eta$ is a shape-dependent constant equal to 0.28 for spheres, globules and spherical endcaps, 0.56 for cylinders, and 1.0 for lamellae, $v_{\mathrm{o}}$ is the molecular volume of a tail, and $R$ is the radius of the sphere or cylinder or half-bilayer thickness. This effective area available for two unconnected chains is not available for the two tails of the gemini molecule because of their connectivity via the spacer. The ratio between the effective area for unconnected chains and the area allowed by the spacer for the connected chains is taken as a measure of the additional packing restriction imposed by the spacer. For a spacer containing $s$ methylene groups, the center-to-center distance of head groups is $(s+1) \ell_{\mathrm{CH}_{2}}$, and this is used to calculate the area allowed by the spacer. On this basis, we write the following expression for the additional packing free energy

$\frac{\left(\Delta \mu_{g}^{\circ}\right)_{\text {pack }}}{k T}=\ln \left[\frac{a_{\text {eff }}}{\left((s+1) \ell_{\mathrm{CH}_{2}}\right)^{2}}\right]$

This free energy contribution is largest for spherical micelles, less for cylindrical micelles, and yet smaller for lamellar aggregates. When $[(s+$ 1) $\left.\ell_{\mathrm{CH}_{2}}\right]^{2} \geq a_{\text {eff }}$, the spacer is long enough to allow the two tails to have conformations equivalent to those of unconnected chains and hence, this contribution ceases to be relevant. 


\subsection{Ionic free energy}

The electrostatic contribution to the free energy in the case of ionic surfactants, $\left(\Delta \mu_{g}^{\circ}\right)_{\text {ionic }}$ has been calculated based on an approximate analytical solution to the Poisson-Boltzmann equation [28].

$$
\begin{aligned}
\frac{\left(\Delta \mu_{g}^{\circ}\right)_{\text {ionic }}}{k T}= & 2\left[\ln \left(\frac{S}{2}+\left[1+\left(\frac{S}{2}\right)^{2}\right]^{1 / 2}\right)\right. \\
& -\frac{2}{S}\left(\left[1+\left(\frac{S}{2}\right)^{2}\right]^{1 / 2}-1\right) \\
& \left.-\frac{2 C}{\kappa S} \ln \left(\frac{1}{2}+\frac{1}{2}\left[1+\left(\frac{S}{2}\right)^{2}\right]^{1 / 2}\right)\right]
\end{aligned}
$$

where

$S=\frac{4 \pi e^{2}}{\varepsilon \kappa a_{\delta} k T}$

The area per head group, $a_{\delta}$ is evaluated at a distance $\delta$ from the hydrophobic core surface, where the counterion is centered [16,17], and $\kappa$ is the inverse Debye screening length. The first two terms on the right hand side of Eq. (14) are the exact solution to the Poisson-Boltzmann equation for planar geometry, while the third term represents a correction for the curvature of the micelle. In the curvature correction term, $C$ depends on the geometry of the aggregate and equals $2 /(R+\delta)$ for spheres, globules and endcaps of cylinders, $1 /(R+\delta)$ for cylinders and zero for lamella.

When the spacer is long, the distance between the two charged head groups of one molecule can be equal to the distance between the charges located on neighboring molecules, i.e. the charges are uniformly distributed over the aggregate surface. In this case, Eq. (14) is satisfactory. However, when the spacer is short, the two ionic groups of a gemini molecule are forced to be closer to one another than they are to the charged groups of adjacent molecules, resulting in the overall distribution of charges at the micellar surface becoming non-uniform. Such a change in surface charge distribution occurs when the extended length of the spacer $s \ell_{\mathrm{CH}_{2}}$ is less than the mean thermodynamic distance between head groups $a^{1 / 2}$. The non-uniformity in charge distribution has two differing consequences, not ac- counted for by Eq. (14). First, since the nearest charges on the neighboring molecules are farther apart compared to the uniform charge distribution case, the actual electrostatic repulsion energy is reduced by the non-uniform charge distribution. Second, the increased concentration of charges locally in the non-uniform charge distribution case will lead to greater condensation of counterions in the proximity of the head groups. This further reduces the electrostatic repulsive energy in the case of the nonuniform charge distribution. These effects are, at present, impossible to estimate quantitatively. Therefore, we adopt a semi-empirical correction factor $F$ to account for the non-uniformity effect and calculate the electrostatic interaction energy to be $(1-F)\left(\Delta \mu_{g}^{\circ}\right)_{\text {ion }}$, with $F$ given by

$F=\left(1-\frac{s \ell_{\mathrm{CH}_{2}}}{a^{1 / 2}}\right)^{0.9} \lambda$

The functional form of the correction factor, $1-$ $\frac{s \ell_{\mathrm{CH}_{2}}}{a^{1 / 2}}$, is suggested by the dependence of Coulombic interaction energy on the distance between the charges $\left(s \ell_{\mathrm{CH}_{2}}\right.$ for the non-uniform case compared to $a^{1 / 2}$ for the uniform case). It is then scaled to account for the curvature of the micelle surface using the ratio $\lambda$ between the electrostatic free energies calculated for spherical (or cylindrical) and planar systems assuming uniform charge distributions.

$\lambda=\frac{\left(\Delta \mu_{g}^{\circ}\right)_{\text {ionic, curved }}}{\left(\Delta \mu_{g}^{\circ}\right)_{\text {ionic, planar }}}$

The exponent 0.9 in Eq. (16) is an empirical constant that is added to qualitatively represent the counterion condensation effect. The value of this exponent cannot be estimated at present on any theoretical grounds. Here, the choice is arbitrary, but with the constraint that this exponent is used as a universal constant for all gemini surfactants irrespective of the tail lengths, spacer lengths, and spacer chemical structures. When $s \ell_{\mathrm{CH}_{2}} \geq a^{1 / 2}$, the charges are uniformly distributed, and $F=0$. 


\section{Molecular constants and computational scheme}

Estimates of molecular constants appearing in the above free energy expressions are available from our earlier work on conventional surfactants [16]. These include the tail volume, $v_{\mathrm{s}}$, the extended tail length, $l_{\mathrm{s}}$, the hard core area of the head group, $a_{\mathrm{p}}$, and the distance $\delta$ from the core surface at which the counterions are located for ionic surfactant aggregates. The area $a_{\mathrm{p}}$ for trimethyl ammonium bromide head groups is taken to be $0.54 \mathrm{~nm}^{2}$, the value used to perform predictive calculations for single chain surfactants [29].

Calculations for molecules of the type: $\left[\left(\mathrm{CH}_{3}\right)_{2}-\right.$ $\mathrm{C}_{m} \mathrm{H}_{2 m+1}-\mathrm{N}^{+}-\left(\mathrm{CH}_{2}\right)_{2}-X-\left(\mathrm{CH}_{2}\right)_{2}-\mathrm{N}^{+}-\mathrm{C}_{m} \mathrm{H}_{2 m+1}-$ $\left.\left(\mathrm{CH}_{3}\right)_{2}\right] 2 \mathrm{Br}^{-}$, where $\mathrm{X}=\mathrm{CH}_{2}, \mathrm{NCH}_{3}, \mathrm{O}$, or S are also carried out in this paper for tail lengths of $m=5-16$. The free energy model given above was used in the calculations for this series of molecules. In principle, the presence of different functional groups could affect the transfer free energy of the spacer, the interfacial tension between the spacer and the aggregate core, and the length of the spacer. We anticipate the role of this functional group to be minor since it is only one unit in a polymethylene chain. Therefore, in all cases, the contribution of the functional group to the length of the spacer is taken to be equal to that of a $\mathrm{CH}_{2}$ unit and the other molecular constants are kept the same as for a polymethylene spacer. Calculations are also performed for molecules with rigid spacers consisting of an aromatic ring, Ar, of the form $\left[\left(\mathrm{CH}_{3}\right)_{2}-\mathrm{C}_{m} \mathrm{H}_{2 m+1}-\mathrm{N}^{+}\right.$ $\left.-\mathrm{CH}_{2}-\mathrm{Ar}-\mathrm{CH}_{2}-\mathrm{N}^{+}-\mathrm{C}_{m} \mathrm{H}_{2 m+1}-\left(\mathrm{CH}_{3}\right)_{2}\right] 2 \mathrm{Br}^{-}$. The spacer length in this case is estimated by taking the aromatic ring $\mathrm{Ar}$ to be equivalent to 5 methylene units. Further, because of its rigidity, the spacer is not allowed to be inside the micelle core, and $s_{\text {core }}=0$. The interfacial tension between the spacer and the micelle core, $\sigma_{\mathrm{sp}}$ is taken equal to zero as for the polymethylene spacer.

The computational scheme has been described previously $[16,17]$. For spherical or globular micelles, where the size distribution is usually narrow, it is assumed that the number (or weight) -average aggregation number, $g_{\mathrm{n}}\left(\right.$ or $g_{\mathrm{w}}$ ) can be taken as the value of $g$ for which $X_{g}$ (or $g X_{g}$ ) is a maximum. The average aggregation number of spherical or globular micelles reported here corresponds to a maximum in $X_{g}$. For cylindrical micelles, the free energy corresponding to the endcaps and the cylindrical part are minimized and the sphere-to-rod transition parameter $K$ is calculated using Eq. (3). The aggregation number at any total surfactant concentration is then calculated using Eq. (4). For planar lamellae, the free energy per molecule is minimized. The free energies of all aggregate shapes are compared at a given value of $X_{1}$ and the shape corresponding to the lowest free energy is taken to be the equilibrium structure.

\section{Results and discussion}

\subsection{Critical micelle concentration}

The predicted CMC values compare reasonably with experimental data as a function of alkyl tail chain length for the $m-3-m, 2 \mathrm{Br}^{-}$bis(quaternary ammonium bromide) gemini surfactants with methylene group spacers (Fig. 1). One can observe that the CMCs of gemini surfactants are substantially smaller than those of the corresponding

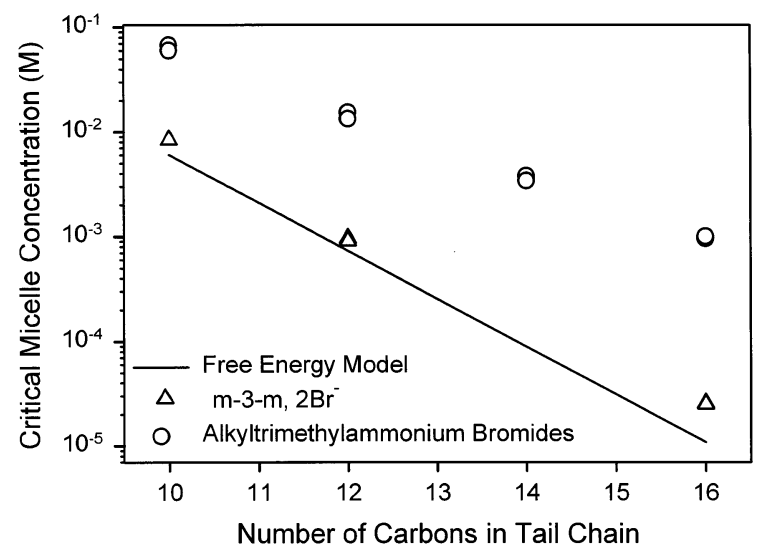

Fig. 1. Model predictions and experimental values [8,31] of CMCs for $m-3-m, 2 \mathrm{Br}^{-}$gemini surfactants. For comparison, $\mathrm{CMC}$ values of alkyltrimethylammonium bromide surfactants are also shown [32,33]. All results correspond to $25^{\circ} \mathrm{C}$. 


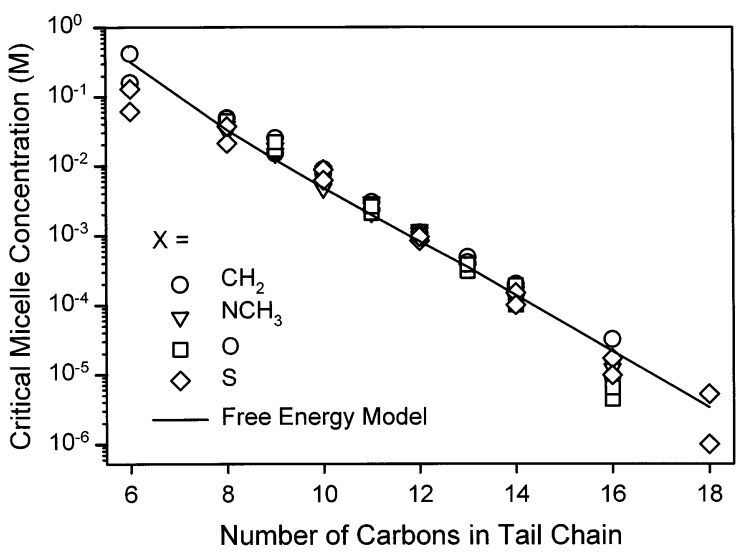

Fig. 2. The effect of surfactant tail length on CMC for $m-5-m, 2 \mathrm{Br}^{-}$surfactants. Experimental data from Ref. [3]. All results correspond to $25^{\circ} \mathrm{C}$.

monomeric analogs, alkyl trimethyl ammonium bromides. The $\mathrm{CMC}$ values are also pre-dicted well for molecules of the type $\left[\mathrm{C}_{m} \mathrm{H}_{2 m+1}-\mathrm{N}^{+}\right.$. $\left.\left(\mathrm{CH}_{3}\right)_{2}-X-\left(\mathrm{CH}_{2}\right)_{2}-\mathrm{N}^{+}-\left(\mathrm{CH}_{3}\right)_{2}-\mathrm{C}_{m} \mathrm{H}_{2 m+1}\right] 2 \mathrm{Br}^{-}$, when $X$ is either $\mathrm{CH}_{2}, \mathrm{NCH}_{3}, \mathrm{O}$, or $\mathrm{S}$, over a wide range of tail chain lengths (Fig. 2). As mentioned earlier, in performing the calculations, the functional group is taken to be equivalent to that of a $\mathrm{CH}_{2}$ unit for all cases, as if the surfactant is an $m-5-m, 2 \mathrm{Br}^{-}$surfactant. Indeed, there is little dependence of the measured CMC values on the different functional groups [3].

For molecules with an aromatic ring in the spacer, agreement between predictions and measurements is satisfactory for tail lengths of 8,10 , and 12. For longer chains, the predicted CMC is significantly lower than the experimental CMC. One may note that the chain length dependence of the experimental CMC undergoes a sudden change at a tail length of 14 whereas the model does not contain any effect to cause such a sudden change. Song and Rosen [30], who obtained the CMC values from surface tension measurements, observed that the surface tension did not lower as much as was expected and the CMCs were higher than expected and nearly constant for tail lengths of 14, 16, and 18 carbon atoms (Fig. $3)$. They attributed the anomalous behavior to the formation of small, non-surface active aggregates. In our model calculations, $\left(\Delta \mu_{g}^{o}\right)_{\mathrm{tr}}$ decreases from
$-34.76 \mathrm{kT}$ when there are 14 carbon atoms to $-43.65 \mathrm{kT}$ when there are 18 carbon atoms. This is the main reason for the significant lowering of the CMC with increasing tail length. It is possible that in estimating the transfer free energy, the group contributions may have to be reduced in magnitude for the long tail molecules. This is because the long tails may be more coiled (compared to short tails) in the monomeric state leading to greater intramolecular contacts within the tail. Such an effect would lead to a lower magnitude for the transfer free energy compared to what has been employed here and to an increase in the predicted CMC. However, as shown in Figs. 1 and 2, for bis(quaternary ammonium bromide) surfactants, the free energy model does closely predict $\mathrm{CMC}$ values when the tails contain 16 and 18 carbon atoms. Hence, explanations other than that based on transfer free energy are warranted for rationalizing the experimental $\mathrm{CMC}$ for molecules with the aromatic ring spacer. It would also be useful to determine the CMC for these molecules via other techniques to examine the validity of this anomalous experimental result.

\subsection{Origin of very low CMCs}

In the framework of the free energy model, the very low $\mathrm{CMC}$ of the gemini surfactants can be directly traced to the larger magnitude of the

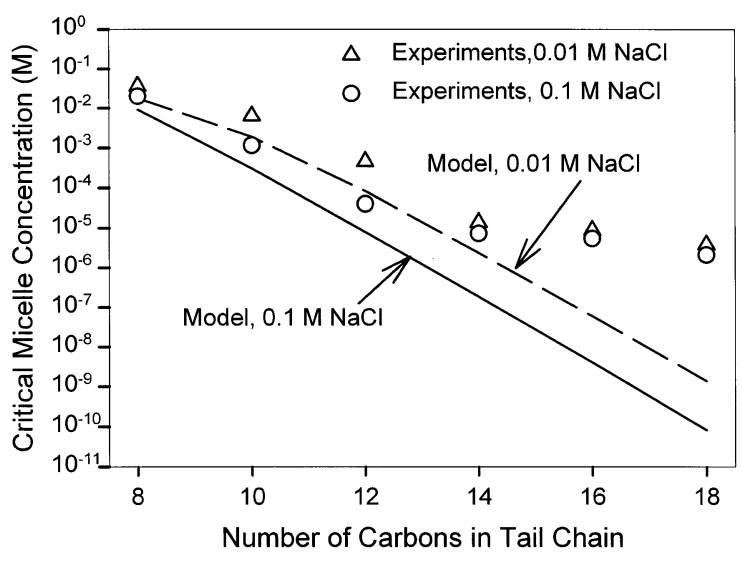

Fig. 3. CMC values for bis(quaternary ammonium bromide) surfactant molecules with aromatic spacers. Data from Ref. [30]. All results correspond to $30^{\circ} \mathrm{C}$. 


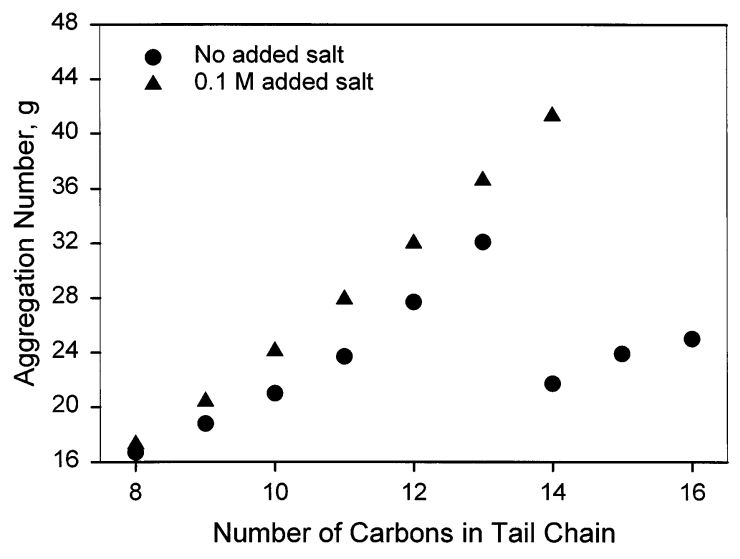

Fig. 4. Aggregation numbers as a function of the number of carbons in the tail, with and without added salt, for $m-5-m, 2$ $\mathrm{Br}^{-}$surfactants. All results correspond to $25^{\circ} \mathrm{C}$.

transfer free energy coming from both the tails. The buried part of the spacer can also contribute to this transfer free energy when the spacer is long, although none of the molecules examined in the present study had spacers long enough to permit a buried portion ( $s_{\text {core }}$ in Eq. (6) was always zero). The CMC of a gemini surfactant depends upon the free energy per molecule and not upon the free energy per chain. Therefore, even if the free energy per chain of a gemini surfactant is comparable to that of its monomeric analog, the free energy per molecule is significantly lower for the geminis, implying a much lower CMC.

\subsection{Size and shape of micelles}

For molecules of the type $\left[\mathrm{C}_{m} \mathrm{H}_{2 m+1}-\mathrm{N}^{+}\right.$$\left.\left(\mathrm{CH}_{3}\right)_{2}-X-\left(\mathrm{CH}_{2}\right)_{2}-\mathrm{N}^{+}-\left(\mathrm{CH}_{3}\right)_{2}-\mathrm{C}_{m} \mathrm{H}_{2 m+1}\right] 2 \mathrm{Br}^{-}$ (treated as equivalent to $m-5-m, 2 \mathrm{Br}^{-}$), spherical or globular aggregates are predicted to form. The aggregation numbers reported in Fig. 4 correspond to a total surfactant concentration of 50 $\mathrm{mM}$. The aggregation numbers do not change with changing surfactant concentration which implies that they are narrowly dispersed [17]. Although slightly smaller micelles are predicted to form for longer tail lengths, the variation in the magnitude of the aggregation numbers over all tail lengths is minor. The aggregation number increases as the number of carbon atoms in the tail increases over the range of 8-13 carbon atoms and then also for tail lengths of 14, 15, and 16 carbon atoms. The discontinuity in the micelle size as a function of tail length can be explained by the fact that the equilibrium size of the aggregates is strongly dependent on the solution ionic strength. Since the CMC values decrease with increasing tail length, in the absence of any added salt, the low monomer concentration determines the ionic strength. This condition leads to larger ionic head group repulsions, and hence limited growth of aggregates for longer tail lengths. Indeed, for ionic single chain surfactants, $g$ usually increases with increasing tail length up to a certain point, and then levels off. Adding some salt into the solution can eliminate this behavior, as can be seen in the calculations for $0.1 \mathrm{M}$ added salt. In this case, the electrostatic repulsions between the head groups diminish, allowing somewhat larger aggregates to form at longer tail lengths.

The formation of large, elongated aggregates when the spacer is short is also predicted by the free energy model. Experimental data and the predictions from the free energy model are in close agreement for 10-3-10,2 $\mathrm{Br}^{-}$and 12-3-12,2

Table 1

Sphere-to-rod transition parameter and shape of micelles

\begin{tabular}{|c|c|c|c|c|c|}
\hline Molecule & $K$ (model) & $\begin{array}{l}K \\
\text { (experiments) }\end{array}$ & Shape (model) & Shape (experiments) & $\begin{array}{l}\text { References for experi- } \\
\text { mental data }\end{array}$ \\
\hline $10-3-10$ & $2.36 \times 10^{5}$ & $1.21 \times 10^{5}$ & Cylinder & Cylinder & {$[31]$} \\
\hline $12-3-12$ & $2.40 \times 10^{6}$ & $4.20 \times 10^{5}$ & Cylinder & Threadlike & {$[9,12,13]$} \\
\hline $16-3-16$ & $1.53 \times 10^{8}$ & $\begin{array}{l}\text { No data } \\
\text { available }\end{array}$ & Cylinder & $\begin{array}{l}\text { Vesicles, bilayer membranes fragments, } \\
\text { threadlike }\end{array}$ & [12] \\
\hline
\end{tabular}


Table 2

Individual contributions to the overall free energy of aggregation for various shapes

Free energy contribution for each shape (kT)

\begin{tabular}{|c|c|c|c|c|}
\hline & & & & \\
\hline & $\left(\Delta \mu_{g}^{o}\right)_{\mathrm{sph}}$ & $\left(\Delta \mu_{g}^{o}\right)_{\mathrm{cyl}}$ & $\left(\Delta \mu_{g}^{o}\right)_{\mathrm{cap}}$ & $\left(\Delta \mu_{g}^{o}\right)_{\mathrm{lam}}$ \\
\hline \multicolumn{5}{|l|}{ A. Molecule: $12-5-12,2 \mathrm{Br}^{-}$} \\
\hline Electrostatic & 7.75 & 8.56 & 7.52 & 12.28 \\
\hline Steric & 1.62 & 1.49 & 1.36 & 1.59 \\
\hline Interfacial & 11.33 & 11.94 & 12.63 & 11.47 \\
\hline Transfer & -31.73 & -31.73 & -31.73 & -31.73 \\
\hline Deformation of tails & 2.58 & 1.77 & 1.98 & 0.94 \\
\hline Coverage & -2.11 & -2.11 & -2.11 & -2.11 \\
\hline Packing & 0.00 & 0.00 & 0.00 & 0.00 \\
\hline Total free energy & -10.57 & -10.08 & -10.35 & -7.57 \\
\hline Area per head group $\left(\mathrm{nm}^{2}\right)$ & 0.67 & 0.69 & 0.73 & 0.66 \\
\hline Radius or half-bilayer thickness (nm) & 1.67 & 1.07 & 1.38 & 0.55 \\
\hline \multicolumn{5}{|l|}{ B. Molecule: $12-3-12,2 \mathrm{Br}^{-}$} \\
\hline Electrostatic & 6.04 & 6.70 & 6.13 & 8.64 \\
\hline Steric & 1.88 & 1.94 & 1.80 & 2.08 \\
\hline Interfacial & 10.45 & 10.29 & 10.69 & 9.96 \\
\hline Transfer & -31.73 & -31.73 & -31.73 & -31.73 \\
\hline Deformation of tails & 2.72 & 2.06 & 2.37 & 1.07 \\
\hline Coverage & -0.70 & -0.70 & -0.70 & -0.70 \\
\hline Packing & 0.62 & 0.33 & 0.75 & 0.42 \\
\hline Total free energy & -10.71 & -11.12 & -10.70 & -10.26 \\
\hline Area per head group $\left(\mathrm{nm}^{2}\right)$ & 0.64 & 0.63 & 0.65 & 0.64 \\
\hline Radius or half-bilayer thickness $(\mathrm{nm})$ & 1.67 & 1.12 & 1.47 & 0.57 \\
\hline
\end{tabular}

$\mathrm{Br}^{-}$(Table 1). Elongated rods or threadlike micelles form, with $K$ values $\sim 10^{5}-10^{6}$. For 16-3$16,2 \mathrm{Br}^{-}$, cryo-TEM imaging revealed vesicles, bilayer membrane fragments, and threadlike micelles [12], whereas the model predicts rodlike aggregates with $K \sim 10^{8}$.

The particular micelle shape that is predicted to form can be rationalized by comparing the individual free energy contributions for various aggregate shapes. As an illustrative example, we compare the individual free energy terms for spherical (12-5-12,2 $\mathrm{Br}^{-}$) and cylindrical (12-312,2 $\mathrm{Br}^{-}$) equilibrium aggregates (Table 2). The transfer free energy and the coverage free energy do not depend on the specific shape of the micelle. Therefore, these contributions need not be considered in explaining why a particular shape forms. For 12-5-12,2 $\mathrm{Br}^{-}$, spherical micelles form and $\left(\Delta \mu_{g}^{o}\right)_{\mathrm{sph}}=-10.57 \mathrm{kT}$, whereas $\left(\Delta \mu_{g}^{o}\right)_{\mathrm{cyl}}=-$ $10.08 \mathrm{kT}$. The ionic repulsion between the head groups and the creation of the micellar interface favor spheres forming compared to cylinders. The steric interactions and the deformation of the tails would tend to make cylindrical micelles favorable. However, the ionic contribution appears to dominate and allow the formation of spheres, providing the largest difference in the individual free energy contributions between sphere and cylinder of $0.81 \mathrm{kT}$.

The molecule with a shorter spacer (12-3-12,2 $\left.\mathrm{Br}^{-}\right)$is predicted to form cylindrical micelles (Table 2B). Although the ionic free energy contribution favors spheres over cylinders by $0.66 \mathrm{kT}$, the interfacial free energy, tail deformation free energy, and tail packing free energy shift the most favorable shape toward cylinders. The tail deformation free energy is an important factor, with a difference of $0.66 \mathrm{kT}$ between $\left(\Delta \mu_{g}^{o}\right)_{\mathrm{def}, \mathrm{sph}}$ and $\left(\Delta \mu_{g}^{o}\right)_{\text {def, cyl }}$.

An interesting case where the model does not completely predict the shapes observed is 16-3$16,2 \mathrm{Br}^{-}$. The free energy model predicts only 
threadlike or cylindrical micelles, while a combination of vesicles, membrane fragments, and threadlike micelles were observed using cryoTEM [12]. For this molecule, the difference between $\left(\Delta \mu_{g}^{o}\right)_{\text {lam }}$ and $\left(\Delta \mu_{g}^{o}\right)_{\mathrm{cyl}}$ is about $1 \mathrm{kT}$, with values of -14.41 and $-15.37 \mathrm{kT}$, respectively. The electrostatic repulsion predicted by the model for the lamella is too high in comparison to the repulsion experienced by a molecule in the infinite cylinder, with a difference of about $2.4 \mathrm{kT}$ between $\left(\Delta \mu_{g}^{o}\right)_{\text {ion, lam }}$ and $\left(\Delta \mu_{g}^{o}\right)_{\text {ion, cyl }}$. This example allows us to see the sensitivity of the electrostatic correction factor in predicting a given micelle shape. If the exponent in the electrostatic correction factor is changed from 0.9 to 0.5 , then the difference in the ionic repulsion terms between cylinder and lamella is only about $1 \mathrm{kT}$, and this reduction is more than enough to allow vesicles to form.

\subsection{Influence of free energy contributions on aggregation}

The calculated individual contributions to the free energy are compared as functions of the micelle aggregation number to determine which terms favor micellar growth and which terms limit their growth (Fig. 5). For all surfactants, the formation of aggregates is made possible by the negative transfer free energy. In terms of absolute magnitude, the transfer energy is the largest free energy term for all the molecules studied. Since the transfer free energy does not depend on the size of the micelle, it does not influence the equilibrium shape of the aggregate. The only other negative contribution to the free energy is provided by the coverage term, which is much smaller in comparison to the transfer free energy. The coverage term is also independent of the size of the aggregate.

The free energy of formation of the micelle core-water interface is a large positive term. It decreases as the micelle size increases and hence, this term favors the growth of micelles. The extra packing free energy term introduced for gemini surfactants (to account for the packing constraint on the tails as they are connected by the spacer) also favors micelle growth since it decreases as micelle size increases. The remaining free energy contributions (ionic and steric repulsions between the head groups, the tail deformation energy) limit the growth of micelles since they increase in magnitude as the micelle size increases.

The ionic term is typically large in magnitude and varies as a function of spacer length. This contribution is smaller for short spacers and increases as the spacer becomes longer. The origin of this effect has been attributed in our model to the non-uniformity of charge distribution at mi-
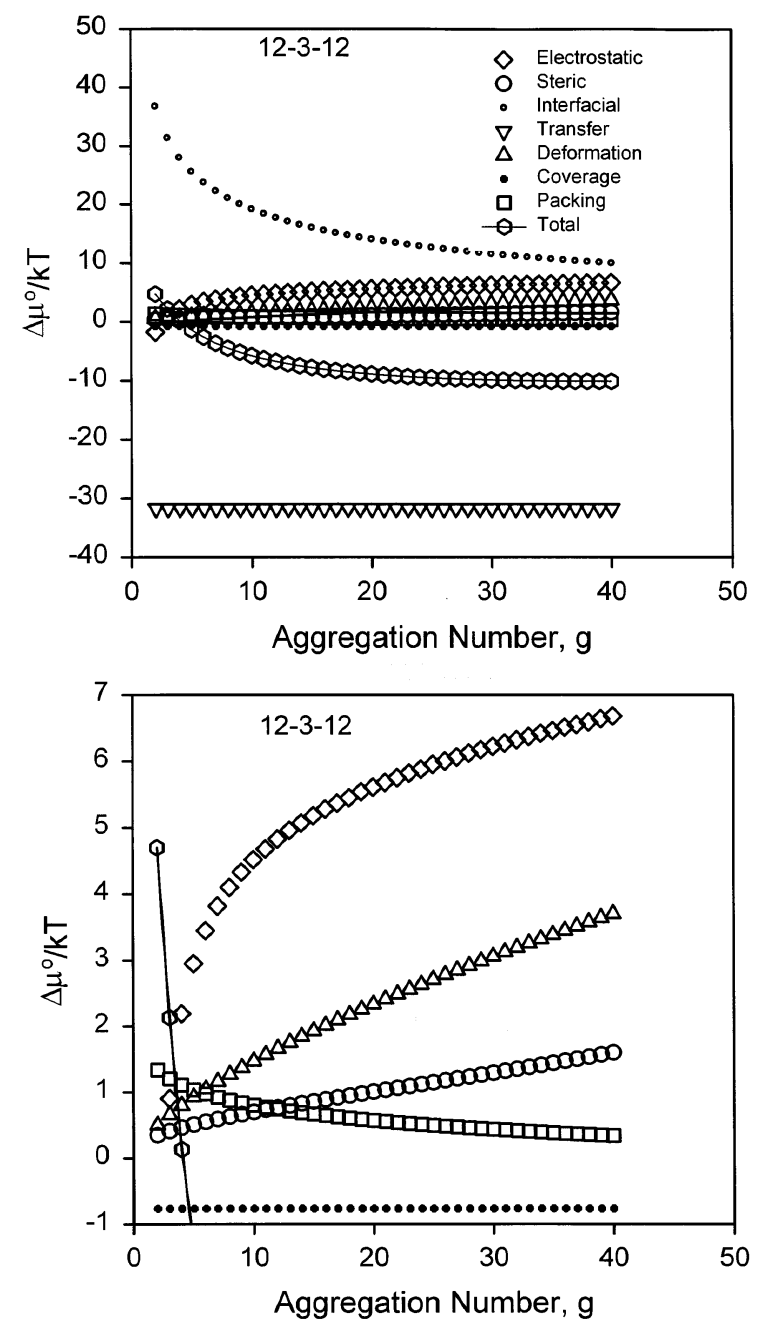

Fig. 5. The dependence of individual free energy contributions on micelle aggregation number for 12-3-12,2 $\mathrm{Br}^{-}$surfactant. The top figure shows all the contributions, while the bottom figure shows an expanded view of some contributions. 
celle surface when the spacer is short, and also the enhanced counterion condensation that will result. As described before, when the spacer is short, the charges at the micellar surface are not evenly distributed because the spacer forces charged head groups of a molecule to be closer together compared to the distance of separation between two different molecules dictated by equilibrium considerations. The magnitude of the electrostatic repulsions are higher when the charges are uniformly distributed, which is the case when the spacer is long enough. For short spacers, the diminished electrostatic repulsion between head groups allows larger micelles to form, such as the threadlike and wormlike structures observed experimentally. The exact form of the empirical correction factor employed for the electrostatic repulsion term plays an important role in the model predictions.

Our modeling suggests that the ionic interaction energy and the deformation of the surfactant tails are the dominant terms in deciding the size and shape micelle that will form. Although these free energy contributions are important in determining the size and shape of conventional ionic surfactants, as well, the properties of gemini surfacants make these two interaction energies more important because (i) the ionic interaction energy has a lower magnitude when the spacer is short because of the nonuniform distribution of charges; and (ii) the extra packing contribution arising from the connectivity of the tails via the spacer decreases as the aggregate curvature decreases (from spheres to cylinders and to lamellae). The coverage term, which is also related to the spacer, while not affecting size and shape, does lower the CMC by making aggregation favorable at a lower concentration. The remaining spacer-dependent contribution, namely the transfer free energy associated with the buried portion of the spacer, was not relevant for the illustrative calculations carried out in this paper since in all cases considered, $s_{\text {core }}$ is zero.

We are extending our free energy model to include a wider range of spacer lengths, anionic and nonionic gemini surfactants, and the solubilization properties of these molecules, as well. This future work will hopefully lead to a more thorough understanding of the micellization and solubilzation properties of gemini surfactants.

\section{Conclusions}

A free energy model is developed in this study to predict micellization properties of gemini surfactants, such as the CMC, micelle aggregation number and the aggregate shape. The model is an extension of the one formulated earlier for conventional surfactants and contains only molecular constants, with one exception. The nonuniform distribution of electrostatic charges at the aggregate surface that occurs for gemini surfactants with short spacers is accounted for empirically, employing however, a common expression for all gemini surfactants. The model is able to predict the CMC values for gemini surfactants with varying tail lengths and different chemical structures of the spacer, such as the presence of $\mathrm{CH}_{2}, \mathrm{NCH}_{3}$, $\mathrm{O}, \mathrm{S}$, and aromatic rings. The predicted size and shape of aggregates generally agrees with evidence from cryo-TEM and other sources. A comparison of the sign, magnitude and shape dependence of the individual contributions to the free energy shows that the nonuniform charge distribution at the aggregate surface and the additional packing constraint imposed on the tails, both due to short spacers, play a central role in determining the equilibrium shapes of the aggregates. The possible formation of threadlike micelles and vesicles/bilayer fragments in the case of gemini surfactants in contrast to the formation of spheres by their monomeric analogs can be directly traced to these two free energy terms.

\section{References}

[1] F.M. Menger, C.A. Littau, J. Am. Chem. Soc. 113 (1991) 1451.

[2] R. Zana, J. Colloid Interface Sci. 78 (1980) 330.

[3] F. Devinsky, I. Lacko, F. Bittererova, L. Tomeckova, J. Colloid Interface Sci. 114 (1986) 314.

[4] M.J. Rosen, L. Liu, J. Amer. Oil Chem. Soc. 73 (1996) 885.

[5] M.J. Rosen, L.D. Song, J. Colloid Interface Sci. 179 (1996) 261. 
[6] R. Zana, Structure-performance relationships in surfactants (Chapter 6), in: K. Esumi, M. Ueno (Eds.), Surfactant Science Series, vol. 70, Marcel Dekker, New York, 1997.

[7] M.J. Rosen, Chemtech 23 (1993) 30.

[8] R. Zana, M. Benrrauo, R. Rueff, Langmuir 7 (1991) 1072.

[9] E. Alami, H. Levy, R. Zana, Langmuir 9 (1993) 940.

[10] N.M. van Os, F.R. Haak, L.A.M. Rupert, Physicochemical Properties of Selected Anionic, Cationic and Nonionic Surfactants, Elsevier, Amsterdam, 1993.

[11] S. De, V.K. Aswal, P.S. Goyal, S. Bhattacharya, J. Phys. Chem. 100 (1996) 11664.

[12] D. Danino, Y. Talmon, R. Zana, Langmuir 11 (1995) 1448.

[13] R. Zana, Y. Talmon, Nature 362 (1993) 228.

[14] H. Diamant, D. Andelman, Langmuir 10 (1994) 2910.

[15] H. Diamant, D. Andelman, Langmuir 11 (1995) 3605.

[16] R. Nagarajan, E. Ruckenstein, Langmuir 7 (1991) 2934.

[17] R. Nagarajan, Structure-performance relationships in surfactants (Chapter 1), in: K. Esumi, M. Ueno (Eds.), Surfactant Science Series, vol. 70, Marcel Dekker, New York, 1997.

[18] C. Tanford, The Hydrophobic Effect, Wiley, New York, 1973.
[19] J.N. Israelachvili, J.D. Mitchell, B.W. Ninham, J. Chem. Soc. Faraday Trans. II. 72 (1976) 1525.

[20] R. Nagarajan, E. Ruckenstein, J. Colloid Interface Sci. 91 (1983) 500.

[21] P. Mukerjee, J. Phys. Chem. 73 (1969) 2054.

[22] P. Mukerjee, J. Phys. Chem. 76 (1972) 565.

[23] P. Mukerjee, J. Pharm. Sci. 68 (1974) 972.

[24] P. Mukerjee, in: K.L. Mittal (Ed.), Solubilzation and Microemulsions, Plenum Press, New York, 1977.

[25] R. Nagarajan, J. Colloid Interface Sci. 90 (1982) 477.

[26] R. Nagarajan, E. Ruckenstein, J. Colloid Interface Sci. 60 (1977) 221.

[27] A.N. Semenov, Soviet Phys. JETP 61 (1985) 733.

[28] D.F. Evans, B.W. Ninham, J. Phys. Chem. 61 (1983) 904.

[29] R. Nagarajan, C. Wang, J. Colloid Interface Sci. 178 (1996) 471.

[30] L.D. Song, M.J. Rosen, Langmuir 12 (1996) 1149.

[31] H. Hirata, N. Hattori, M. Ishida, H. Okabayashi, M. Frusaka, R. Zana, J. Phys. Chem. 99 (1995) 17778.

[32] N.A. Klimenko, T.V. Karmazina, N.A. Yaroshenko, A.E. Bartnitskii, Zh. M. Aryamova, Kolloidnyi Zhurnal. 46 (1984) 1112.

[33] P.J. Sams, E. Wyn-Jones, J. Rassing, Chem. Phys. Lett. 13 (1972) 233. 